\title{
Impacto de la investigación dentro del proceso de formación profesional
}

\section{Impact of research within the process of professional training}

\author{
DOI: http://dx.doi.org/10.17981/cultedusoc.11.2.2020.09
}

Recibido: 3 de abril de 2020 Aceptado: 25 de junio de 2020 Publicado: 09 de julio de 2020

Nubia Estella Cruz Casallas

Corporación Universitaria Minuto de Dios - UNIMINUTO. Villavicencio (Colombia) nubia.cruz@uniminuto.edu

Numar Álvarez Cardona

Universidad Nacional Abierta y a Distancia - UNAD. Acacias (Colombia) numar.alvarez@unad.edu.co

Lenith Cárdenas Torrado

Corporación Universitaria Minuto de Dios -UNIMINUTO. Villavicencio (Colombia) lenith.cardenas@uniminuto.edu

Para citar este artículo:

Cruz, N., Álvarez, N. y Cárdenas, L. (2020). Impacto de la investigación dentro del proceso de formación profesional. Cultura, Educación y Sociedad, 11(2). 145-160. DOI: http://dx.doi.org/10.17981/cultedusoc.11.2.2020.09

\section{Resumen}

Las Instituciones de Educación Superior (IES) están llamadas a formar profesionales competentes, capaces de transformar la sociedad con ideas innovadoras y factibles que se ajusten a un ambiente incierto y cambiante, de modo que se satisfagan las necesidades, se promueva la participación y se favorezca la equidad social. En este contexto, el presente artículo de revisión tiene como propósito analizar los avances y aportes relacionados con el proceso de investigación en las Instituciones de Educación Superior, a través de la descripción de los tipos de investigación, indicadores y metodologías para la medición del impacto dentro del proceso de formación profesional. La información presentada fue extraída de documentos publicados entre los años 2003 y 2018, escritos en español e inglés, los cuales fueron seleccionados atendiendo a su grado de pertinencia teórico-conceptual, relevancia y contenido. Para el procesamiento de la información se empleó la técnica de análisis de contenido, a través de la cual se estudió la información recolectada y se abstrajeron los principales conceptos, teorías y aportes realizados por diferentes exponentes en el área de estudio. El trabajo realizado permitió evidenciar el valor de las labores de investigación que adelantan las IES y su impacto en la formación profesional; de allí la necesidad de formular y ejecutar proyectos bajo un enfoque transdisciplinar, para establecer mejores vínculos y trabajar coordinadamente con la comunidad y el sector productivo, en aras de realizar transferencia de conocimiento y lograr la solución de problemas en un contexto global fluctuante y competitivo. Palabras clave: Investigación; formación profesional; competencias

\begin{abstract}
Higher Education Institutions (HEIs) are called to train competent professionals, capable of transforming society with innovative and feasible ideas that adjust to an uncertain and changing environment, so that needs are met, participation is promoted and favored social equity. In this context, the purpose of this review article is to analyze the advances and contributions related to the research process in Higher Education Institutions, through the description of the types of research, indicators and methodologies for measuring the impact within of the professional training process. The information presented was extracted from documents published between 2003 and 2018, written in Spanish and English, which were selected based on their degree of theoretical-conceptual relevance, relevance and content. For the information processing, the content analysis technique was used, through which the collected information was studied and the main concepts, theories and contributions made by different exponents in the study area were abstracted. The work carried out made it possible to demonstrate the value of the research work carried out by HEIs and their impact on professional training; hence the need to formulate and execute projects under a transdisciplinary approach, to establish better links and work in coordination with the community and the productive sector, in order to carry out knowledge transfer and achieve problem solving in a fluctuating and competitive global context.
\end{abstract}

Keywords: Research; professional development; competencies 


\section{INTRODUCCIÓN}

El proceso social del conocimiento incluye, no solo la producción o generación del nuevo conocimiento, sino además los mecanismos que se deben desarrollar para la efectiva difusión, aplicación y transferencia de los mismos. Es por esta razón que las instituciones de Educación Superior (IES), como espacios de formación profesional, dan un lugar preponderante a la ciencia, no solo por el hecho de ser el espacio donde se asimila el conocimiento en sus diferentes disciplinas, sino porque dentro de su quehacer, hay producción de nuevo conocimiento a través de los ejercicios investigativos que desarrollan los profesores y estudiantes y porque esos mismos conocimientos, deberían en su derecho, ir dirigidos a la transformación del entorno social donde se desenvuelve la institución (Fabre, 2005).

De acuerdo a Erazo (2013), la Organización de las Naciones Unidas para la Educación, la Ciencia y la Cultura-UNESCO en su declaración mundial sobre la educación superior en el siglo XXI: visión y acción realizada en el año de 1998, proclamó que las tres funciones sustantivas de las IES son: Docencia, Investigación y Proyección Social; desde éstas se busca la formación de sujetos integrales, con capacidad para resolver problemáticas de su entorno a través de la aplicación creativa del conocimiento. Por lo anterior, es necesario evaluar en qué medida las acciones de las tres funciones son pertinentes, y cómo y cuándo los egresados, las investigaciones, los programas de extensión o las experiencias obtenidas tienen aplicabilidad en la sociedad (Parra y Arias, 2016). Por lo anterior, para medir el impacto de las acciones de una IES en la formación de sus estudiantes, se deberán tener en cuenta los resultados, los efectos y el tiempo (Libera, 2007).

El rol de la investigación y el impacto que ésta genera son objeto de discusión debido a que aún no existen criterios fiables ni rigurosos que permitan obtener certezas científicas de sus efectos (Ortiz, Viamontes y Reyes, 2015). Al respecto, la evaluación sobre la pertinencia y efectividad es cada vez más necesaria por cuanto se hace necesario identificar a tiempo las acciones de mejora que permitan encaminar el ejercicio investigativo hacia una formación profesional alineada con los requerimientos actuales.

\section{Metodología}

Se realizó una revisión de literatura relacionada con el tema de estudio en repositorios de universidades y bases de datos como Scopus, Redalyc, Google Académico, entre otras. La búsqueda se limitó a documentos publicados entre 2003 y 2018, escritos en español e inglés, los cuales fueron seleccionados atendiendo a su grado de pertinencia teórico conceptual, relevancia y contenido. Para el procesamiento de la información se empleó la técnica de análisis de contenido, abarcando las etapas de pre-análisis, exploración del material, organización e interpretación, proceso que permitió estudiar la información recolectada y abstraer los principales conceptos, teorías y aportes realizados por diferentes exponentes en el área de la investigación en Instituciones de Educación Superior. Al respecto Marín-González, Cabas, Cabas y Paredes-Chacín (2018), señalan que a través de esta técnica se abstraen categorías, cuyo tratamiento desde la lógica del pensamiento, permite trascender la realidad mediante la descripción de atributos y cualidades. 


\section{Resultados DEL ANÁlisis DE LOS REFERENTES TEÓRICOS}

\section{La investigación en los procesos de formación Universitaria}

La investigación, el desarrollo tecnológico y la innovación son categorías que influyen de forma determinante en el crecimiento científico, económico y social de una comunidad; de ahí que Albornoz, Estébanez y Alfaraz (2005) afirmen que "El análisis del impacto social de la ciencia y la tecnología se conecta de modo directo, como ha sido señalado, con la política científica y tecnológica, aunque de un modo indirecto también con la política social" (p. 75). Lo expuesto en la cita anterior, no viene siendo otra cosa que la responsabilidad de la universidad como ente investigador frente al desarrollo de la sociedad; papel que se asume en el "momento en que la producción se transformó en una función de la ciencia y el trabajo científico tomó el mando en el impulso al progreso de las fuerzas productivas" (Figueroa, 2013, p. 11).

De igual forma, estos mismos autores indican que, a nivel global, el papel de las instituciones de Educación Superior está ligado a cuatro funciones básicas: la generación de nuevo conocimiento para la producción; la obtención de personal calificado bien sea para producción, servicios o investigación; la generación de los cuadros para la conducción económica, social y política de los países y la producción de ideología, incluida la crítica orientada al mejoramiento del estado de cosas en la sociedad. En tal sentido Touraine expresa:

El crecimiento es el resultado, más que de la acumulación de capital solamente, de un conjunto de factores sociales. Lo más nuevo es que depende mucho más directamente que antes del conocimiento, y, por consiguiente, de la capacidad de la sociedad para crear creatividad (Albornoz et al., 2005, p. 76).

Con este argumento se refuerza la obligatoriedad de la investigación frente a los procesos sociales, al desarrollo comunitario y sobre todo a la profundización y ampliación del conocimiento. Al respecto, Rodelo y Castro (2016) destacan la importancia de los procesos formativos para el desarrollo humano y social del individuo.

En la actualidad se espera y se le exige a la universidad y a sus investigadores que asuman un nuevo papel en el desarrollo de las propuestas de crecimiento académico, en donde la capacidad de sus investigadores para la selección, valoración y análisis de información mediado dentro de un espacio temporal son claves para su éxito (Fandos, 2006), buscando obtener resultados verdaderamente útiles y de impacto social (Milanés, Solís y Navarrete, 2010); es decir, que la investigación proveniente de los claustros universitarios responda a las verdaderas necesidades cotidianas.

Consecuente con lo anterior, el primer objetivo de las IES para la formación en investigación de los futuros profesionales, es garantizar la formación de competencias generales, tales como pensamiento crítico, rigor científico y autonomía intelectual, gestión de la innovación, gestión de proyectos, entre otros; para luego articular estas competencias a las prácticas investigativas dirigidas por docentes investigadores (Guerrero, 2007). Prácticas investigativas que en sus lógicas deben ir direccionadas a la solución de problemáticas específicas. 
A lo anterior se suma la necesidad constante de mantener relación estrecha con el entorno, de tal manera que el ejercicio mismo de producción de nuevo conocimiento sea pertinente, innovador y responda a las necesidades presentes; es decir que tenga alto impacto en el bienestar social, lo cual necesariamente conlleva a lo que se ha denominado como "desarrollo social". En este sentido, Davyt y Mujica (2013) estudiaron las distintas formas de apoyo y encontraron que en la última década han aparecido mecanismos de apoyo al desarrollo social y menciones en los planes nacionales de ciencia, tecnología e innovación, con lo cual se está respondiendo al llamado que la investigación debe enfrentar como responsable de aportar al crecimiento integral de la sociedad.

Al respecto, Escobar, Castaño, Ruiz y Restrepo (2016) manifiestan la necesidad de que la comunidad académica ejecute procesos investigativos colaborativos, buscando la participación activa de docentes y estudiantes, de modo que se ofrezcan espacios para la reflexión y transformación de los procesos, proyectos y productos de la labor investigativa, con el objetivo de realizar ajustes y avanzar hacia una verdadera evaluación de impacto social, contribuyendo de esta manera a la solución de problemas y a la construcción de un nuevo orden social.

En este sentido se pone en evidencia el valor conceptual y contextual de la pertinencia social de las actividades investigativas de las IES, toda vez que para la producción de conocimiento se parte de procesos de innovación, buscando generar bienestar y calidad de vida, desarrollo socio-económico y cambios en el seno mismo de la ciencia y la tecnología, por lo que las IES deben orientarse por lineamientos de políticas nacionales y en concordancia con un plan estratégico de desarrollo de acciones nacionales, regionales y locales (Colina, 2007).

Consecuentemente las IES deben orientar su accionar en concordancia con las políticas nacionales y sociales, de tal forma que se promueva la participación activa de la comunidad y el sector productivo, procurando la generación de nuevos saberes por medio de la innovación, el trabajo coordinado y la difusión de los resultados alcanzados con el desarrollo de las investigaciones; el propósito es satisfacer las necesidades de la humanidad, contribuyendo al enriquecimiento y modificación de paradigmas que han prevalecido en determinados contextos sociohistóricos.

\section{Tipos de Investigación en las Instituciones de Educación Superior}

Para las instituciones de educación superior, asumir la investigación como propósito es un reto y es parte de su esencia (Guerrero, 2007). En tal sentido, su actividad científica se ha orientado hacia el criterio de la pertinencia de su accionar frente a los cambios impuestos por el desarrollo científico, tecnológico y de innovación (CTI), al incidir en las transformaciones epistemológicas, tecnológicas y pedagógicas en la sociedad global, en el contexto de la transdisciplinariedad y diversidad (Colina, 2007). En este sentido, el ejercicio investigativo de las IES está basado en el desarrollo de la investigación básica y de la investigación aplicada, desde diferentes niveles y contextos.

La investigación básica se fundamenta en la generación de teorías científicas a partir de una idea o de la constatación de un fenómeno físico, por lo que es fundamental para el avance del conocimiento. Por su parte, la investigación aplicada es la encargada de 
enlazar la teoría con el producto, siendo su objetivo la generación de conocimiento con aplicación directa y a mediano plazo en la sociedad o en el sector productivo (Lozada, 2014). En este sentido la investigación aplicada ejerce un valor preponderante por su capacidad para trascender en la sociedad.

Como una necesidad para mejorar el ejercicio de la investigación aplicada, surge la investigación endógena la cual no es más que una investigación etnográfica en la que los investigadores, orientados por expertos y/o asesores, hacen parte de la población investigada, participando en el diseño mismo de la investigación en cuanto a objetivos, focos de interés, métodos y marco referencial (Martínez, 2006). Este tipo de investigación es considerada valiosa en el trabajo con comunidades cuya estructura y manejo social es complejo, permitiendo mejorar su incidencia social en cuanto a la aplicabilidad y sostenibilidad de las acciones implementadas.

En general, se considera que los proyectos de investigación tienen que generar impacto en el medio donde se desarrollan; por ello una de las principales acciones es la socialización de los resultados de la investigación por parte de los investigadores, en los sitios donde se realizaron los proyectos y ante la comunidad científica, lo cual se realiza a través de conferencias, talleres o socialización de memorias. De igual forma se espera que este ejercicio investigativo genere a mediano y largo plazo, un efecto curricular en los programas de formación (Martínez, 2013).

\section{Responsabilidad Social en la investigación de las IES}

En el marco de la Conferencia Mundial de Educación Superior, desarrollada por la UNESCO (2009), se manifiesta la dificultad para mantener un buen equilibrio entre la investigación básica y la aplicada; debido a los altos niveles de inversión que necesita el primer tipo de investigación y el reto que representa vincular el conocimiento de ámbito mundial con los problemas locales. En atención a lo expuesto, se establece como un principio de responsabilidad social la promoción de la ciencia y la interdisciplinariedad, al servicio de la sociedad. Igualmente, en la conferencia se declara:

La educación superior tiene la responsabilidad social de hacer avanzar nuestra comprensión de problemas polifacéticos con dimensiones sociales, económicas, científicas y culturales, así como nuestra capacidad de hacerles frente. La educación superior debería asumir el liderazgo social en materia de creación de conocimientos de alcance mundial para abordar retos mundiales, entre los que figuran la seguridad alimentaria, el cambio climático, la gestión del agua, el diálogo intercultural, las energías renovables y la salud pública (UNESCO, 2009, p. 2).

Por otra parte, González, Chirinos, Faría, Olivero y Boscán (2015) manifiestan la importante misión que tiene la universidad en la generación de conocimientos para su permanente actualización y modernización, de manera que se logre dar respuesta a los problemas sociales del entorno y se alcance la integración de factores internos como la democratización del ingreso, calidad educativa y factores externos como la pertinencia de los productos, mejoramiento de mecanismos de financiamiento, entre otros. 
Por lo anterior, combinar la ciencia, tecnología y la responsabilidad social en la educación de las futuras generaciones permitirá formar jóvenes no solo con un alto nivel de conocimientos, sino también con un enorme compromiso social y un deseo ferviente por descubrir y dar respuestas a los problemas que aquejan a la humanidad.

\section{Impacto social en el ejercicio de la investigación}

El impacto es definido como el efecto favorable o desfavorable que produce una acción o actividad en determinado tiempo (Rodríguez y Cobas, s.f.). Al respecto Pichardo (como se citó en González y Calcetero, 2009) define el impacto social como los cambios deseados en el contexto social en que se inscriben las políticas, programas y proyectos que se ven reflejados en las variables en cuanto a la satisfacción de las necesidades básicas y no básicas, la promoción de condiciones para el mejoramiento de la calidad de vida y de trabajo, la generación, fortalecimiento o consolidación de las formas de expresión y participación y el fomento de cambio de actitudes, aptitudes y mentalidad de los actores sociales.

El impacto social también puede ser definido como todo aquello que origina afectación en la calidad de vida de las personas y las comunidades (Ayuso, 2018), atendiendo a lo expresado por Groves (citado en Daza et al., 2017) a la diferencia entre una línea base y los cambios generados en esta, luego de una intervención determinada. En este sentido Kostoff (citado por Fernández, s.f.) señala que el impacto de la investigación es el cambio realizado sobre la sociedad debido al producto de la misma.

Para Lozano, Saavedra y Fernández (2011), el impacto de la ciencia y tecnología, es evaluado en tres aspectos: en el conocimiento, en lo económico y en lo social. Para el impacto del conocimiento se utilizan los índices bibliométricos, en y desde la comunidad científica, ya que este no puede ser medido directamente sino a través de las expresiones del conocimiento como papers, patentes y estudiantes formados, lo cual puede proveer una imagen incompleta del producto del proceso investigativo. El proceso anterior es conocido como cienciometría, la cual ha contribuido a la identificación de indicadores para la gestión de la política científica y tecnológica y en los procesos de toma de decisiones estratégicas (Arencibia y De Moya, 2008).

El segundo aspecto, el impacto económico, utiliza indicadores económicos que miden la incidencia de la innovación en la productividad y competitividad de las empresas; mientras que el tercero, el impacto social, debido a su complejidad aún carece de metodologías estandarizadas para su medición. Sin embargo, utiliza en alguna medida los aspectos anteriores, además de otras variables definidas por la misma comunidad científica, ya que se hace necesario que su análisis se realice desde y en la comunidad científica, entre los mismos agentes generadores y consumidores de conocimiento científico (Milanés et al., 2010).

Por lo anterior, la efectividad de la investigación se puede entender como el grado de focalización sobre las metas que se desean alcanzar, teniendo en cuenta que mientras algunos impactos pueden ser tangibles, otros son intangibles, presentando dificultad en su identificación y cuantificación. Ahora bien, según el Interorganizational Committee on Principles and Guidelines for Social Impact Assessment (Twigg, 2007) el impacto 
social puede ser entendido como las consecuencias originadas sobre las poblaciones humanas derivadas de cualquier acción pública o privada que pueda alterar el modo en que las personas viven, trabajan, se divierten, establecen relaciones, se organizan y en términos generales la forma de reaccionar como miembros de la sociedad. De esta manera se confirma lo expuesto por Libera Bonilla (2007), quien apoya la relación causa-efecto generada por los resultados de dicho proyecto a mediano y largo plazo, los cambios verificados en los grupos o comunidades y la sostenibilidad de los cambios presentados (positivos o negativos).

\section{Evaluación del impacto social (EIS)}

Albornoz et al. (2005) expresan la importancia de analizar el impacto de la ciencia y la tecnología en la sociedad ya que, a partir de esta medición, se inicia un proceso de reflexión en torno a la potencialización o corrección de los efectos favorables o los no deseados; igualmente permite la evaluación de la pertinencia del financiamiento brindado a la actividad científica e innovadora. La medición del impacto social debe ir más allá de la evaluación de la eficacia, permitiendo realizar análisis de los efectos generados sobre la población intervenida (Libera, 2007); es decir, posibilitar la evaluación de los comportamientos desencadenantes generados por los efectos en el desarrollo de las acciones investigativas (Ojeda, 2010).

El estudio del impacto social del conocimiento puede realizarse atendiendo a tres niveles: macro, meso y microsocial, cada uno de los cuales presenta interés para la observación de diversas dimensiones, por lo que las particularidades metodológicas deben ser tenidas en cuenta al momento de abordar el análisis. Sin embargo, es necesario tener en cuenta que la evaluación del impacto social en las IES presenta algunas limitaciones, definidas específicamente en deficiencias conceptuales y metodológicas en los investigadores, en las escasas experiencias que se encuentran sistematizadas sobre evaluación de impacto social que permitan ser tomadas como referentes y, específicamente, en la carencia de políticas y directrices institucionales que establezcan el proceso de evaluación de impacto como requisito (Escobar et al., 2016).

El impacto se mide constatando los resultados y colocándolos en correlación con la intención inicial. No obstante, es válido aclarar que la evaluación por impacto se asocia, no con los resultados propiamente dichos como pudiera considerarse, sino con los beneficios o efectos de dichos resultados (Lozano et al., 2011). Para Fernández, Peláez y Figueredo (2014) el concepto de impacto social debe incluir no sólo los resultados previstos sino también aquellos que no se previeron.

Moñux et al. (2006) manifiestan que la finalidad de la EIS de un proyecto I+D puede dividirse en tres componentes:

- Representar una fuente de información adicional a los socios del proyecto con el objetivo de mejorar el impacto social de los bienes y servicios generados a partir del desarrollo de dicho proyecto. 
- Contribuir al mejoramiento del grado de conciencia entre los gestores de actividades de I+D+I sobre los efectos de los bienes y servicios producto del proyecto ejecutado y la utilidad de la EIS.

- Extender la relevancia de la evaluación de impacto social entre los responsables de los proyectos.

Atendiendo a Twigg (2007), la EIS puede ser entendida como el proceso de análisis, seguimiento y gestión de las consecuencias sociales relacionadas con políticas, programas y proyectos, las cuales pueden ser positivas o negativas, intencionadas o no, directas o indirectas; a su vez pueden ser efectos a corto o largo plazo. Adicionalmente, la EIS contribuye a explicar el cambio en la calidad de vida de las personas, indicando las alternativas que podrían plantearse para mitigar los cambios perjudiciales o promover los beneficiosos.

Según Briones (citado en González y Calcetero, 2009), la evaluación del impacto social es considerada como una forma de investigar, razón por la cual debe ser asumida como proceso ya que se encuentra direccionada a la obtención de un conocimiento científico sobre la estructura, transformaciones y cambios de la realidad social. En este evaluar el impacto social, tendrá como propósito, determinar con los actores locales si el proyecto produjo los cambios sociales establecidos en los objetivos e indicadores que se habían formulado para con ello, definir estrategias para hacerlo más pertinente y sustentable, asegurando la implementación de los cambios.

De acuerdo a Morales y Roux (2015), la evaluación de impacto social tiene como objetivo principal el "establecer las acciones de prevención, mitigación y control de los impactos negativos que sobre los grupos humanos relacionados, puedan ocasionar las afecciones ambientales de actividades de desarrollo" (p. 114). Atendiendo a lo expresado por MacPherson (citado en Morales y Roux, 2015) los objetivos específicos de la EIS son los siguientes:

- Prevenir que el proyecto degrade la calidad de vida de los residentes en las áreas que tendrá incidencia el proyecto.

- Evitar la generación de impactos negativos indirectos por causa de consecuencias sociales no anticipadas derivadas de las acciones del proyecto.

- Reducir la posibilidad de conflictos entre los promotores, interesados e involucrados y el gobierno.

- Identificar áreas en las cuales la gestión del proyecto pueda desarrollarse de manera eficiente y con costos menores.

- Lograr que los beneficios obtenidos por el desarrollo del proyecto, excedan los costos totales.

- Prevenir la generación de costos indirectos por causa del desarrollo del proyecto.

- Evitar que los riesgos generados por el proyecto afecten las poblaciones vulnerables.

- Lograr que el proyecto no afecte los procesos de cohesión e identidad social. 


\section{Indicadores del impacto social}

Rondón (2004) expresa que los indicadores del impacto de la ciencia y tecnología permiten a los países realizar una comparación entre la inversión realizada y la producción científica, buscando diferenciar los años de gestión y obtener información útil para evaluar la relevancia y cuantía de la investigación científica, proponiendo los siguientes indicadores para reflejar los impactos producto y servicio de la ciencia y la tecnología en la sociedad:

\section{Productos}

- Cantidad de proyectos que generan impactos socialmente medibles en campos como la educación, la vivienda, el ambiente, la salud, el trabajo, entre otros.

- Inversiones y personal vinculado en el proyecto.

- Número y clase de grupos de trabajo conformados.

- Número de localidades que han sido impactadas y estimación de poblaciones vinculadas.

- Clase de impacto predominante en cualquiera de los campos social, económico, laboral, ambiental, político o educativo.

- Monto invertido en los proyectos que generan impacto.

\section{Servicios}

- Número de indicadores relacionados con el desarrollo del proyecto que presentan cambios apreciables.

- Cambios porcentuales reflejados en los indicadores o variables de trabajo del proyecto (expost y exante).

- Descripción cualitativa de los cambios surgidos por la aplicación de los proyectos en los diferentes campos de interés como educación, ambiente, vivienda y trabajo.

- Recomendaciones encaminadas al mejoramiento de la aplicación de los proyectos.

Para la medición del impacto social, Quevedo, Chía y Rodríguez Batista (como se citó en Rodríguez, 2005), establecieron un elemento denominado contribución al desarrollo de la sociedad definido como el sector priorizado en el ámbito del desarrollo social y económico del país, en el cual incurren productos, servicios, procesos y tecnologías que, por acción de la investigación, el desarrollo y la innovación ( $\mathrm{I}+\mathrm{D}+\mathrm{I})$ contribuyen al beneficio y mejoramiento de los siguientes indicadores sociales: Cultura, Educación, Salud, Deporte y recreación, Seguridad alimentaria, Empleo, Calidad de vida, Ideología y Defensa y orden interior. 


\section{Metodologías para la Medición del Impacto Social}

Para el sector social, la medición del impacto representa la necesidad de demostrar el efecto positivo de sus resultados al abordar las diferentes problemáticas sociales. De esta manera, Ebrahim y Rangan (2010) compilan las principales metodologías para medición del impacto social utilizadas por instituciones del orden internacional, entre las que se encuentran: rendimiento esperado, ensayos aleatorios controlados, marco lógico, outcomes de la gestión, cuadros de mando, mapas de estrategia y herramientas relacionadas, informes de percepción, evaluación basada en la historia y cambios significativos, evaluación rural participativa y variantes, evaluación participativa de la situación de pobreza, sistemas organizativos para la planificación del impacto, evaluación, rendición de cuentas y aprendizaje y ciencia de la complejidad y enfoque de pensamiento sistémico. En este análisis sobresale la metodología empleada por la Social Return on Investment (SROI), con la que se busca medir el retorno de la inversión social, facilitando la gestión interna y la comparación con otras empresas sociales (Díaz, Marcuello y Marcuello, 2012).

Para la medición del impacto social de la ciencia y la tecnología, Díaz et al. (2012) identifican tres enfoques. El primer enfoque se centra en la evaluación respecto a las políticas de ciencia y tecnología. El segundo en el análisis de correlación entre indicadores sociales e indicadores de ciencia y tecnología buscando hacer comparaciones entre países. El último enfoque evalúa respecto al uso, difusión y efecto del conocimiento generado en el campo social y a partir de sus usuarios y beneficiarios. En todo caso, toda metodología para la medición de impacto social debe desarrollarse en tres etapas: (1) identificación y valoración a nivel interno en los primeros 6 meses de vida (fase ex-ante), (2) evaluación en los primeros dos años a las estrategias implementadas para afrontar los impactos socioeconómicos negativos identificados en la fase ex-ante (fase in-itinere) y la (3) evaluación al final de proyecto para evaluar la aplicabilidad del nuevo conocimiento, es decir, para su efectividad en la aplicación (fase ex-post) (Moñux et al., 2006; Rodríguez y Cobas, s.f.). De igual forma Mendizábal, Gómez y Moñux (2003) sostienen que la metodología de evaluación debe concentrarse un número determinado de fases concretas que cubren todo el ciclo de vida del proyecto.

Según Ortegón, Pacheco y Prieto (2005), la metodología de marco lógico es una herramienta usada en la conceptualización, diseño, ejecución y proceso de evaluación de los proyectos; se encuentra orientada hacia grupos de beneficiarios y posibilita la participación y comunicación entre los involucrados. Puede emplearse en la totalidad de las fases del proyecto: identificación y valoración de actividades, en el diseño de los proyectos de forma sistemática y lógica, valoración de los proyectos, implementación, revisión y evaluación del progreso de los proyectos. La herramienta presenta la siguiente estructura metodológica: análisis de involucrados, análisis de problemas, análisis de objetivos, análisis de alternativas, estructura analítica del proyecto, matriz de marco lógico y evaluación intermedia.

Rodríguez y Cobas (s.f.) identifican a la matriz de marco lógico que se maneja en la formulación y ejecución, como la herramienta fundamental en el proceso de evaluación de un proyecto $\mathrm{I}+\mathrm{D}+\mathrm{I}$, siendo para ellos, el tiempo entre 3 a 5 años posterior a la ter- 
minación del proyecto, como el periodo ideal para hacer la medición. De igual manera estos autores establecen cinco parámetros básicos a tener en cuenta en el proceso de evaluación: pertinencia, eficacia, eficiencia, impacto/propósito y sostenibilidad, los cuales deberán ser evaluados de forma cualitativa (juicio y percepción) y cuantitativa (datos estadísticos) por medio de expertos debidamente seleccionados y a través de indicadores y niveles de calificación establecidos. Establecen igualmente una secuencia metodológica que implica selección de los expertos, selección de proyectos, estudio documental, creación de línea base, evaluación por expertos, elaboración del informe y proceso de socialización de resultados.

Otra de las metodologías empleadas para la evaluación de impacto social es la Matriz de Impactos Cruzados Multiplicación Aplicada a una Clasificación (MICMAC), herramienta que permite el análisis cualitativo de las variables de un sistema dentro de una empresa, sociedad, país y demás contextos, diseñada por Michael Godet en el año 2007 (Garza y Cortez, 2011).

Atendiendo a lo expresado por Arango y Cuevas (2015), el método MICMAC posibilita la identificación de futuros problemas, permitiendo la planeación de estrategias adecuadas.

\section{ConClusiones}

La investigación es la base para el progreso de la humanidad, ya que permite la generación de nuevo conocimiento y el planteamiento de propuestas para la solución de problemas existentes; de allí la necesidad de desarrollar investigación efectiva, de manera que se intervenga activamente en los grupos sociales a modo de promover conductas positivas en la sociedad.

Para el logro de este propósito, las Instituciones de Educación Superior deben fomentar que los docentes investiguen, buscando el enriquecimiento y retroalimentación de su labor; así mismo propender que los estudiantes comprendan la importancia del ejercicio investigativo y de su aplicación durante su vida laboral, contribuyendo a la formación de profesionales competentes y a la generación de oportunidades.

La evaluación del impacto social de la investigación debe realizarse antes, durante y después de finalizado el proyecto, a través de la identificación de indicadores sociales construidos en conjunto con los actores y expertos que intervinieron en su formulación, ejecución y cierre, así como con la población beneficiaria; proceso que permitirá determinar la efectividad de las acciones adelantadas, identificar factores a mejorar y diseñar estrategias acordes a las necesidades actuales, de modo que se contribuya significativamente al fortalecimiento de la educación profesional.

Existen varias metodologías para determinar el impacto social de las investigaciones, como por ejemplo la técnica de rendimiento esperado, ensayos aleatorios controlados, marco lógico, cuadros de mando, mapas de estrategia, informes de percepción, Micmac, entre otras; de allí la importancia de elegir la herramienta adecuada que permita evaluar el efecto causado en la población sujeto de estudio y la identificación de oportunidades de mejora que puedan ser empleadas en futuros proyectos investigativos. 
Las IES deben actuar en concordancia con las políticas nacionales y sociales, promoviendo la participación activa de la sociedad y el sector productivo, procurando la generación de nuevos conocimientos, contribuyendo al enriquecimiento y modificación de paradigmas que han prevalecido en determinados acontecimientos históricos.

En síntesis, el análisis de las referencias seleccionadas permitió evidenciar el valor de las labores de investigación que adelantan las IES y su impacto en la formación profesional; ya que como se registra en la literatura consultada, los egresados que participan en actividades de investigación durante el pregrado, cuentan con las herramientas suficientes para vincularse al desarrollo de proyectos, contribuyendo significativamente a la transformación de la sociedad y al crecimiento económico; de allí la necesidad de formular y ejecutar investigaciones bajo un enfoque transdisciplinar, estableciendo mejores vínculos y trabajando coordinadamente con la comunidad y el sector productivo, en aras de realizar transferencia de conocimiento y lograr la solución de problemas en un contexto global fluctuante y competitivo.

\section{REFERENCIAS}

Albornoz, M. \& Estébanez, M. y Alfaraz, C. (2005). Alcances y limitaciones de la noción de impacto social de la ciencia y la tecnología. Revista Iberoamericana de Ciencia, Tecnología y Sociedad-CTS, 2(4), 73-95. Disponible en http://hdl.handle. net/20.500.12424/54619

Arango, X. A. y Cuevas, V. A. (2014). Método de análisis estructural: matriz de impactos cruzados multiplicación aplicada a una clasificación (MICMAC). [Tesis de maestría]. Universidad Autónoma de Nuevo León, San Nicolás de los Garza, México. Disponible en http://eprints.uanl.mx/6167/

Arencibia, J. \& de Moya, F. (2008). La evaluación de la investigación científica: una aproximación teórica desde la cienciometría. ACIMED, 17(4). Disponible en http:// eprints.rclis.org/11603/

Ayuso, S. (2018). La medición del impacto social en el ámbito empresarial. [Documento de trabajo no. 26]. Barcelona: ESCI-UPF School of International Studies. Recuperado de http://mango.esci.upf.edu/DOCS/Documents-de-treball/26-Medicion-del-impacto-social.pdf

Colina, L. (2007). La investigación en la educación superior y su aplicabilidad social. Laurus, 13(25), 330-353. Disponible en http://revistas.upel.digital/index.php/laurus/article/view/7694

Davyt, A. y Mujica, A. (2013). Resultados de una experiencia de fomento a la investigación e innovación con impacto social en Uruguay. Redes, 19(37), 183-206. Disponible en https://ridaa.unq.edu.ar/handle/20.500.11807/583

Daza, S., Maldonado, O., Arboleda, T., Falla, S., Moreno, P., Tafur, M. y Papagayo, D. (2017). Hacia la medición del impacto de las prácticas de apropiación social de la ciencia y la tecnología: propuesta de una batería de indicadores. História, Ciências, Saúde - Manguinhos, 24(1), 145-164. Disponible en https:/pesquisa.bvsalud.org/ portal/resource/pt/biblio-840688 
Díaz, M. \& Marcuello, C. y Marcuello, C. (2012). Empresas sociales y evaluación del impacto social. CIRIEC-España, Revista de Economía Pública, Social y Cooperativa, (75), 178-198. Recuperado de http://ciriec-revistaeconomia.es/wp-content/uploads/ CIRIEC_7508_Diaz_et_al.pdf

Ebrahim, A. y Rangan, V. K. (2010). The Limits of Nonprofit Impact: A Contingency Framework for Measuring Social Permormance. [Harvard Business School Working Paper, núm. 10-099]. Boston: HBS. Recuperado de https://www.hbs.edu/faculty/Pages/item.aspx?num=37921

Erazo, G. I. (2013). Docencia, investigación y proyección social: Funciones fundamentales de la Universidad. Madrid: Editorial Académica Española.

Escobar, J. V., Castaño, D. A., Ruiz, M. P. y Restrepo, J. C. (2016). Evaluación auténtica del impacto social de procesos, proyectos y productos de investigación universitaria: Un acercamiento desde los grupos de investigación. Revista Lasallista de Investigación, 13(1), pp. 166-180. https://doi.org/10.22507/rli.v13n1a14

Fabre, G. C. (2005). Las funciones sustantivas de la Universidad y su articulación en un departamento docente. $\mathrm{V}$ congreso internacional virtual de educación, Universidad Agraria de la Habana, Cuba. Recuperado de http://sedici.unlp.edu.ar/handle/10915/24694

Fandos, M. (2006). El reto del cambio educativo: nuevos escenarios y modalidades de formación. EDUCAR, 38(1), 243-258. Disponible en https://www.raco.cat/index.php/ Educar/article/view/72357

Fernández, E. (s.f.). La medición del impacto social de la ciencia y la tecnología”. [Online]. Recuperado de www.oei.es/historico/ctsiima/polcuch.pdf_

Fernández, A., Peláez, A. y Figueredo, J. M. (2014). Indicadores sociales para evaluar el impacto del proyecto Ligas Deportivas Comunitarias. EFDeportes, 19(195). [Online]. Disponible en https://www.efdeportes.com/efd195/indicadores-sociales-paraevaluar-el-impacto.htm

Figueroa, V. M. (2013). El rol de la Universidad en el desarrollo. La perspectiva de los organismos internacionales. En, M. E. Martínez, F. J. Piñero y S. A. Figueroa, (Coords.), El papel de la universidad en el desarrollo (pp. 11-22). Buenos Aires y Puebla: Universidad Nacional del Centro de la Provincia de Buenos Aires (UNICEN)/Benemérita Universidad Autónoma de Puebla (BUAP). Disponible en http:// biblioteca.clacso.edu.ar/clacso/se/20140211121020/universidad.pdf

Garza, J. B. y Cortez, D. V. (2011). El uso del método MICMAC y MACTOR análisis prospectivo en un área operativa para la búsqueda de la excelencia operativa a través del Lean Manufacturing. Innovaciones de Negocios, 16(8), 335-356. Disponible en http://eprints.uanl.mx/12560/

González, C. y Calcetero, J. R. (2009). Evaluación del impacto social: una estrategia de investigación para Trabajo Social. Tendencias \& Retos, (14), 43-57. Disponible en http://www.ts.ucr.ac.cr/binarios/revistas/co/rev-co-tendencias-0014-04.pdf

González, M. \& Chirinos, E., Faría, C., Olivero, J. y Boscán, J. (2015). Pertinencia e impacto social de la investigación universitaria en Venezuela. Multiciencias, 15(3),303309. Disponible en http://produccioncientificaluz.org/index.php/multiciencias/article/ view/20976 
Guerrero, M. (2007). Formación de habilidades para la investigación desde el pregrado. Acta Colombiana de Psicología, 10(2), 190-192. Disponible en https://actacolombianapsicologia.ucatolica.edu.co/issue/view/49

Libera, B. (2007). Impacto, impacto social y evaluación del impacto. ACIMED, 15(3). [Online]. Recuperado de bvs.sld.cu/revistas/aci/vol15_3_07/aci05307.htm

Lozada, J. (2014). Investigación Aplicada: Definición, Propiedad Intelectual e Industria. CienciAmérica, 3(1), 47-50. Disponible en http://cienciamerica.uti.edu.ec/openjournal/index.php/uti/article/view/30

Lozano, J., Saavedra, R. y Fernández, N. (2011). La evaluación del impacto de los resultados científicos. Metodologías y niveles de análisis. Humanidades Médicas, 11(1), 99-117. Disponible en http://humanidadesmedicas.sld.cu/index.php/hm/article/ view/23

Marín-González, F., Cabas, L., Cabas, L. y Paredes-Chacín, A. (2018). Formación Integral en Profesionales de la Ingeniería. Análisis en el Plano de la Calidad Educativa. Formación universitaria, 11(1), 13-24. https://dx.doi.org/10.4067/S071850062018000100013

Martínez, M. (2006). Pertinencia social en la investigación endógena. Espacio Abierto, 15(4), 725-740. Disponible en http://produccioncientificaluz.org/index.php/espacio/ article/view/1307/0

Martínez, J. (2013). Responsabilidad social en la investigación aplicada. Investigaciones Andina, 15(26), 625-626. Disponible en https://revia.areandina.edu.co/index.php/ IA/article/view/28

Mendizábal, G. A., Gómez, F. J. y Moñux, D. (2003). Desarrollo de una Guía de Evaluación de Impacto Social para Proyectos de I+D+I. Revista Iberoamericana de Ciencia, Tecnología, Sociedad e Innovación, (5), [Online]. Disponible en https://www.oei. es/historico/revistactsi/numero5/articulo4.htm

Milanés, Y., Solís, F. y Navarrete, J. (2010). Aproximaciones a la evaluación del impacto social de la ciencia, la tecnología y la innovación. Revista Cubana de Información en Ciencias de la Salud, 21(2), 161-183. Disponible en https://www.medigraphic.com/ cgi-bin/new/resumen.cgi?IDARTICULO=30600

Morales, D. \& Roux, R. (2015). Estudio de impacto social: antecedentes y línea base para San Fernando, Tamaulipas. Revista Internacional de Ciencias Sociales y Humanidades, SOCIOTAM, XXV(1), 111-130. Disponible en https://www.redalyc.org/ articulo.oa?id=654/65452535006

Moñux, D., Aleixandre, G., Gómez, F. J., Cáceres, S., Miguel, L. J. y Velasco, E. (junio, 2006). Evaluación del impacto social de proyectos de Investigación y Desarrollo tecnológico (I+D): Una aplicación en el sector de las comunicaciones industriales. I Congreso Iberoamericano de Ciencia, Tecnología, Sociedad e Innovación, CTS+I. México, D.F., México. Recuperado de https://www.oei.es/historico/memoriasctsi/mesa6/ m06p17.pdf.

Ojeda, A. (2010). El Impacto de la Investigación en la Vida Social de los Grupos y las Personas. Psicología Iberoamericana, 18(2), 5-7. Disponible en https://www.redalyc. org/pdf/1339/133915921001.pdf 
Ortegón, E., Pacheco, J. F. y Prieto, A. (2005). Metodología del marco lógico para la planificación, el seguimiento y la evaluación de proyectos y programas. [Online]. Recuperado de https://repositorio.cepal.org/bitstream/handle/11362/5607/S057518_ es.pdf-

Ortiz, E. A., Viamontes, Y. I. y Reyes, N. (2015). La evaluación del impacto científico en las investigaciones educativas a través de un estudio de caso. Revista Electrónica de Investigación Educativa, 17(2), 89-100. Disponible en https://redie.uabc.mx/redie/ article/view/422

Parra, E. y Arias, S. (2016). Metodología para caracterización y análisis de impacto en el medio de egresados de las Instituciones de Educación Superior. Revista Interamericana de Investigación, Educación y Pedagogía, 9(2), 79-96. Disponible en https:// revistas.usantotomas.edu.co/index.php/riiep/article/view/3616

Rodelo, M. y Castro, S. (2016). Reflexiones sobre la educabilidad: elemento fundamental en el proceso educacional del desarrollo humano. Cultura Educación y Sociedad, 7(2), 94-104. Recuperado de https://revistascientificas.cuc.edu.co/culturaeducacionysociedad/article/view/1104

Rodríguez, A. (2005). Impacto social de la ciencia y la tecnología en Cuba: una experiencia de medición a nivel macro. Revista Iberoamericana de Ciencia, Tecnología y Sociedad - CTS, 2(4), 147-171. Disponible en http://www.revistacts.net/files/Volumen\%202\%20-\%20N\%C3\%BAmero\%204/doss05.pdf

Rodríguez, R. y Cobas, M. (s.f.). Metodología de evaluación de impactos de proyectos de investigación. [Online]. Recuperado de https://inis.iaea.org/collection/NCLCollectionStore/_Public/45/078/45078486.pdf_

Rondón, L. (2004). Indicadores del impacto de la ciencia y tecnología (CT) en la sociedad: reflexiones y avances. Espacios, 25(2). Disponible en https://www.revistaespacios. com/a04v25n02/04250222.html

Twigg, J. (2007). Herramientas para la integración de la reducción del riesgo de desastres: Evaluación del Impacto Social. [Online]. Recuperado de http://www.proventionconsortium.net/themes/default/pdfs/tools_for_mainstreaming_GN11-sp.pdf_

UNESCO. (2009). Conferencia Mundial sobre la Educación Superior - 2009. La nueva dinámica de la educación superior y la investigación para el cambio social y el desarrollo. [Online]. Recuperado de http://www.unesco.org/education/WCHE2009/comunicado_es.pdf_

Nubia Estella Cruz Casallas es Ingeniera Agrónoma, Magíster en Acuicultura. Actualmente me desempeño como Directora de Investigación de la Corporación Universitaria Minuto de Dios - UNIMINUTO, Vicerrectoría Regional Orinoquía (Colombia). https:// orcid.org/0000-0002-0979-5095

Numar Álvarez Cardona es Licenciado en Lingüística y Literatura. Magíster en Educación. Laboro como docente en la Escuela de Ciencias de la Educación de la Universidad Nacional Abierta y a Distancia - UNAD (Colombia). https://orcid.org/0000-0001-95597134 
Lenith Cárdenas Torrado es Ingeniera Industrial de la Universidad de Pamplona, Magíster en Ingeniería Industrial de la Universidad Nacional. Desempeño mi labor como docente adscrita a la Dirección de Investigación de la Corporación Universitaria Minuto de Dios - UNIMINUTO, Vicerrectoría Regional Orinoquía (Colombia). https://orcid. org/0000-0002-0189-8153 